\title{
ALOMETRIA DE PLÂNTULAS E JOVENS DE ESPÉCIES ARBÓREAS: COPA X ALTURA
}

\author{
Rita de Cássia Quitete Portela ${ }^{1,3}$ \& Flavio Antonio Maës dos Santos ${ }^{2}$
}

Biota Neotropica v3 (n2) - http://www.biotaneotropica.org.br/v3n2/pt/abstract?article+BN00503022003

\author{
recebido em: 30/04/2003 \\ revisado em: 23/06/2003 \\ publicado em: 01/07/2003
}

\author{
${ }^{1}$ Programa de Pós-Graduação em Biologia Vegetal, Departamento de Botânica, IB, CP 6109, UNICAMP, 13083-970, \\ Campinas, SP, Brasil \\ ${ }^{2}$ Departamento de Botânica, IB, CP 6109, UNICAMP, 13083-970, Campinas, SP, Brasil - www.ib.unicamp.br \\ ${ }^{3}$ Autor para correspondência: rita@ quiteteportela.com.br, Rua José Linhares 17/401, Leblon, Rio de Janeiro, RJ, CEP: \\ 22430-220.
}

\begin{abstract}
Most of the morphological and developmental characteristics of different parts of a plant may vary in function of the species architecture, microenvironment and degree of ecological restriction where its has begun it growth. This study aimed to analyze the allometric relation between height and the largest horizontal crown axis and, for a community of tree seedlings and saplings up to $1 \mathrm{~m}$ in height in the Reserva Estadual do Morro Grande (23o35' S - 23050'S; 46o45'W 47o15'W), situated in São Paulo's Atlantic Plateau, SE Brazil. We sampled 600 individuals in $341 \mathrm{~m}^{2}$ plots distant $2.5 \mathrm{~m}$ from each other along six transects, 100 individuals per transect. For each individual we measured their height and largest crown axis. We adjusted different regression models (linear, exponential, power, polynomial and logarithmic) to the data. The best fit was for the power model $\left(r^{2}=0.640, p<0.001\right)$. The fact that juveniles from different species adjust to the same allometric model suggests that ecological factors could have an important restrictive role in the allometry of seedlings and saplings of tree species.
\end{abstract}

Key words: allometry, seedling, height, crown, Atlantic forest

\section{Resumo}

Muitos caracteres morfológicos e de desenvolvimento de diferentes partes de uma planta variam dependendo da arquitetura da espécie, do microambiente, e do grau de restrição ecológica onde esta iniciou seu crescimento. Dentro deste contexto, este estudo teve como objetivo analisar a relação alométrica entre o maior eixo de copa e a altura, para a comunidade de plântulas e indivíduos jovens de espécies arbóreas com até $1 \mathrm{~m}$ de altura na Reserva Estadual do Morro Grande (23o35'S - 23o50’S; 46o45’W - 47o15’W), situada no Planalto Atlântico de São Paulo. Foram feitas seis transecções onde a cada 2,5 metros foi estabelecida uma parcela de $1 \mathrm{x} 1 \mathrm{~m}$, totalizando 34 parcelas. Todos os indivíduos encontrados dentro das parcelas tiveram a altura e o maior eixo horizontal da copa medidos, sendo amostrados em cada transecção 100 indivíduos, totalizando uma amostra de 600 indivíduos. Foram ajustados diferentes modelos de regressão aos dados (linear, exponencial, potencial, polinomial e logarítmica) sendo que a potencial apresentou o melhor ajuste $\left(r^{2}=0,640, p<0,001\right)$. O fato de jovens de diferentes espécies se ajustarem a um único modelo alométrico sugere que os fatores ecológicos podem ter um papel restritivo na alometria das plântulas e jovens de espécies arbóreas.

Palavras-chave: alometria, plântula, copa, altura, floresta Atlântica

http://www.biotaneotropica.org.br 


\section{Introdução}

Os estudos de alometria em plantas são importantes para a compreensão de aspectos ecológicos e evolutivos em espécies de plantas e os modelos gerados a partir desses estudos podem ser ferramentas poderosas de previsão em ecologia (Bond et al. 1999). O estudo da relação entre tamanho e forma em árvores também é importante no entendimento da estrutura e dinâmica das florestas (King 1996, Sposito \& Santos 2001). Muitos caracteres morfológicos e de desenvolvimento de diferentes partes de um indivíduo, variam dependendo da arquitetura específica, do microambiente e do grau de restrição ecológica onde este iniciou seu crescimento (Kohyama 1987, King 1996, Cornelissen 1999, Dalling et al. 1999, Nishimura \& Suzuki 2001, Wright \& Westoby 2001). Essas relações também variam de acordo com a espécie em questão (Kohyama 1987, Niklas 1994, Bond et al. 1999, Cornelissen 1999, Kidson \& Westoby 2000, Sposito \& Santos 2001, Sterck \& Bongers 2001, Alves \& Santos 2002) e com a fase de desenvolvimento em que a planta se encontra, pois os indivíduos sofrem transformações mecânicas ao longo do seu crescimento (Niklas 1994, Sterck \& Bongers 2001, Alves \& Santos 2002).

Este estudo teve como objetivo analisar a relação alométrica entre o maior eixo de copa e a altura, para a comunidade de plântulas e indivíduos com até $1 \mathrm{~m}$ de altura de espécies arbóreas, em uma Floresta Estacional Semidecidual com elementos da Floresta Ombrófila Densa. A pergunta endereçada neste artigo foi: plântulas e indivíduos pequenos possuem a mesma relação alométrica entre o maior eixo de copa e a altura?

\section{Material e Métodos}

Área de estudo - A Reserva Estadual do Morro Grande (ca. 11000 ha) localiza-se no município de Cotia, nos bordos do Planalto Atlântico de São Paulo (23o35'S - 23o50'S; $\left.46045^{\prime} \mathrm{W}-47 \mathrm{o} 15^{\prime} \mathrm{W}\right)$, encontrando-se protegida há cerca de 80 anos. O tipo de vegetação é de Floresta Estacional Semidecidual com elementos da Floresta Ombrófila Densa (Veloso et al. 1991), sendo constituida de um grande mosaico sucessional, com áreas que já sofreram corte e áreas que nunca foram cortadas. O clima da região é temperado e chuvoso macrotérmico de inverno seco, do tipo Cwa (Köppen 1948, Ometto 1981), com temperatura anual variando de 11 oC a 27 oC e precipitação média anual em torno de $1.400 \mathrm{~mm}$ (SABESP 1997).

Desenho experimental - Para a caracterização da comunidade de plântulas e árvores jovens com até $1 \mathrm{~m}$ de altura (indivíduos pequenos), foram feitas 6 transecções na Reserva, com uma média de distância entre si de 2,1 km. Nenhum indivíduo foi identificado, somente os indivíduos da família Arecaceae foram identificados a nível de família.
Estes foram identificados pois mesmo quando plântulas não deixam qualquer dúvida sobre a sua classificação. Cada transeção teve início a 100 m da estrada principal da Reserva e foi disposta sempre em paralelo a estrada. De 2,5 em 2,5 metros, em cada transecção, foi estabelecida uma parcela de 1x1m. Foram totalizadas 34 parcelas nas 6 transecções, sendo que cada transecção teve um número variável de parcelas. Os 100 primeiros indivíduos pequenos encontrados dentro das parcelas em cada transecção tiveram suas alturas e o maior eixo horizontal da copa medidos, totalizando uma amostra de 600 indivíduos. A altura foi considerada como a distância da superfície do solo até o meristema apical e o maior eixo foi considerado o maior comprimento horizontal da copa. Para a medição do maior eixo horizontal da copa, foi considerado a disposição natural das folhas. Os dados foram coletados com uma régua de $1 \mathrm{~m}$ em junho-julho de 2000.

Foram ajustados diferentes modelos de regressão (linear, exponencial, potencial, polinomial e logarítmica) (Zar 1999) aos dados de altura $(\mathrm{cm}) \mathrm{x}$ maior eixo $(\mathrm{cm})$ para verificar qual descrevia melhor a relação entre as variáveis.

\section{Resultados}

A maioria dos indivíduos amostrados (mediana) tinha até $11,0 \mathrm{~cm}$ de altura, sendo que o menor indivíduo tinha 2,5 cm e o maior $99,0 \mathrm{~cm}$ (figura 1). O tamanho máximo do maior eixo horizontal da copa foi 70,0 cm, e o mínimo de $1,0 \mathrm{~cm}$, com a maioria dos indivíduos (mediana) medindo até $8,0 \mathrm{~cm}$ (figura 2). Menos de $25 \%$ dos indivíduos possuíam altura superior a 25,0 cm e maior eixo horizontal da copa superior a $15,0 \mathrm{~cm}$.

A melhor relação entre maior eixo horizontal da copa (C) e a altura $(\mathrm{H})$ foi potencial $\left(\mathrm{C}=1,250 \mathrm{H}^{0,738}, \mathrm{r}^{2}=0,64, \mathrm{p}<\right.$ 0,001 , figura 3 ), com as plantas de alturas maiores apresentando proporcionalmente menores eixos. Os demais modelos de regressão (linear, exponencial, polinomial e logaritmico) apresentaram menores ajustes $\left(\mathrm{r}^{2}<0,55\right)$. Os indivíduos da família Arecaceae (1\% do total de indivíduos) apresentaram uma relação diferente (pontos mais extremos no gráfico de regressão, figura 3) dos demais indivíduos, onde o maior eixo apresentou um incremento proporcionalmente maior que a altura. Quando estes indivíduos são tirados da análise, a relação entre as duas variáveis continua a mesma, assim como a equação e o coeficiente de determinação .

\section{Discussão}

A maioria dos indivíduos amostrados $(77,2 \%$ do total de indivíduos), foi menor que $0,25 \mathrm{~m}$ de altura, indicando que na Reserva Estadual do Morro Grande existe uma maior 


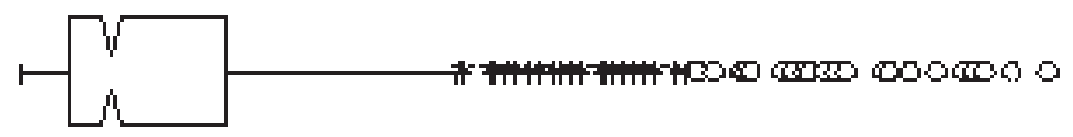

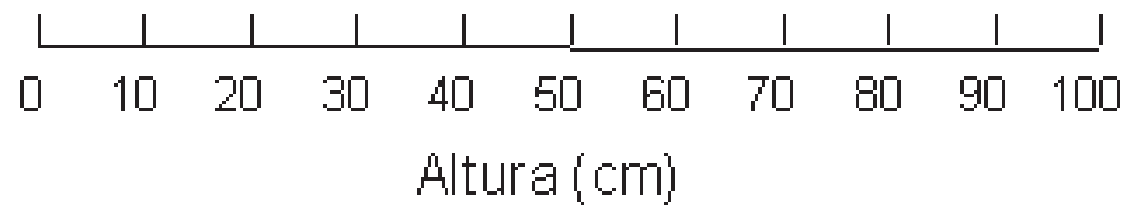

Figura 1: Distribuição de altura dos indivíduos com até $1 \mathrm{~m}$ de altura na Reserva Estadual do Morro Grande. Cada caixa ("box”) representa $50 \%$ das observações, e as barras horizontais inferiores e superiores representam a amplitude total da distribuição (25\% e 75\%), exceto os valores extremos (*) e os muito extremos ( ${ }^{\circ}$ ) ("outliers"). A cintura dentro da caixa representa a mediana, e os limites de confiança de $95 \%$ são representados pelas linhas indentadas ao redor da mediana.

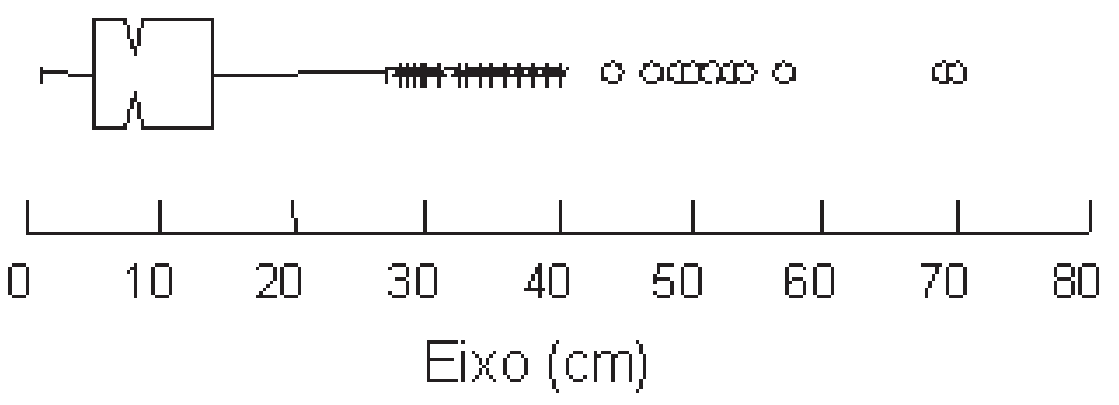

Figura 2: Distribuição de tamanho do maior eixo horizontal da copa dos indivíduos com até 1 m de altura na Reserva Estadual do Morro Grande.

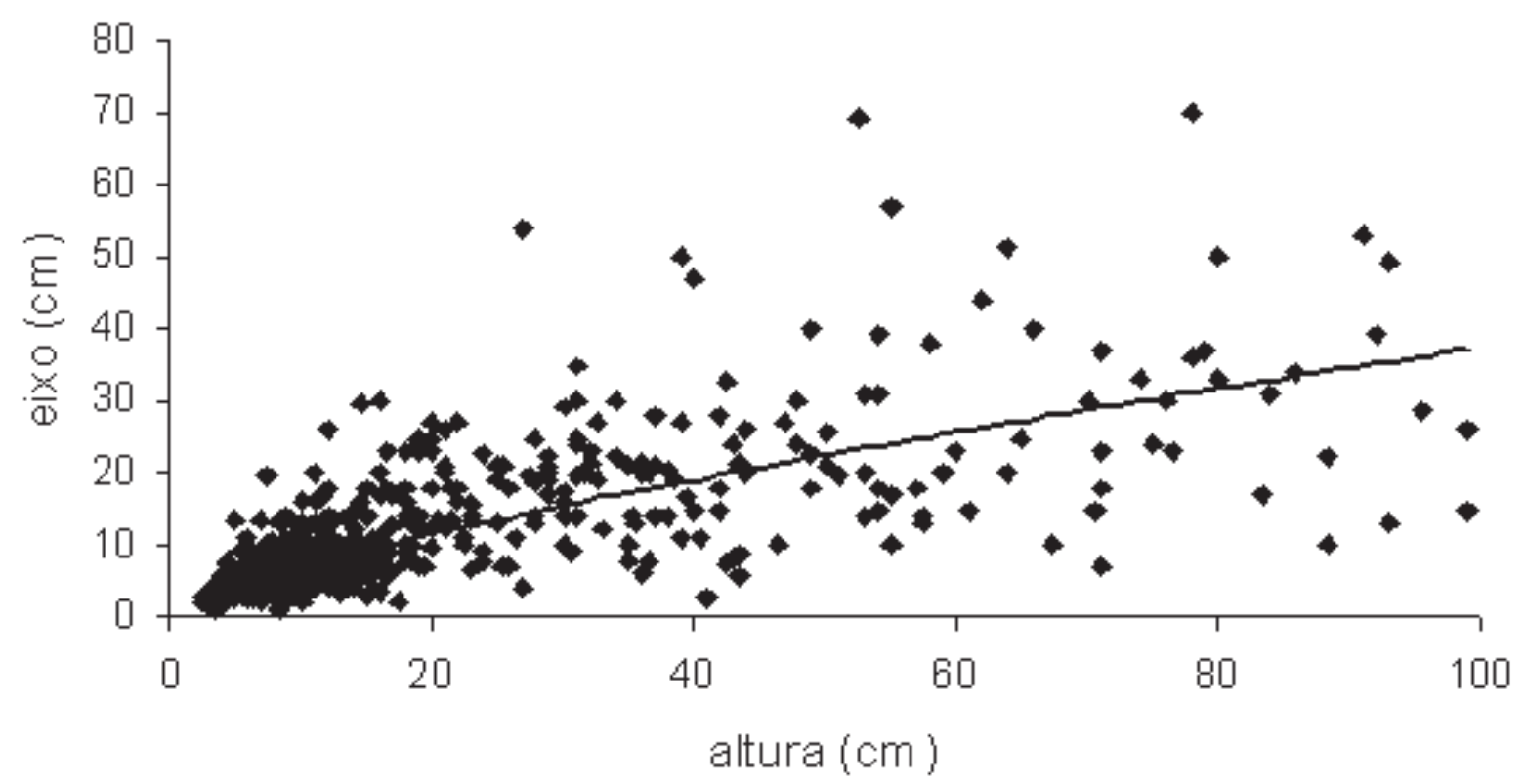

Figura 3: Relação entre maior eixo horizontal da copa $(C$ em $\mathrm{cm})$ e altura $(H$ em $\mathrm{cm})$ de indivíduos de espécies arbóreas com até $1 \mathrm{~m}$ de altura, na Reserva Estadual do Morro Grande, Cotia, $S P .\left(C=1,250 H^{0,738}, r^{2}=0,64, p<0,001\right)$

http://www.biotaneotropica.org.br 
concentração de plântulas, do que de jovens. Considerando que a coleta de dados foi feita durante a estação seca (junhojulho), época não favorável a germinação de sementes, esse acúmulo de indivíduos pequenos nas áreas amostradas não parece ser resultado de um intenso recrutamento de plântulas recém-germinadas quando da coleta de dados, sugerindo a formação de um banco de plântulas e dificuldades no desenvolvimento inicial dessas plântulas. Tais dificuldades podem ser resultantes de pressões ambientais, como pouca luminosidade no interior da floresta, reduzindo as chances de crescimento de indivíduos no sub-bosque da floresta (Canham 1988, King 1994, Sterck \& Bongers 2001, Wright \& Westoby 2001) e, consequentemente, causando a formação de banco de plântulas.

O fato de jovens de diferentes espécies se ajustarem a um único modelo alométrico sugere que os fatores ecológicos podem ser bastante restritivos e terem um importante papel na determinação da alometria de plântulas e jovens na área estudada, principalmente por todas estarem sujeitas à condições ambientais severas como a falta de luz. King (1996) obteve resultado semelhante ao analisar a relação de uma medida de copa com a altura, para 14 espécies arbóreas em uma floresta tropical úmida na Costa Rica. As condições próximas ao solo de florestas tropicais são severas, principalmente com relação as limitações impostas pela baixa incidência de luz, podendo limitar a assimilação e produção de recursos, tornando esse ambiente bastante restritivo ao estabelecimento de novos indivíduos (Kohyama 1987). O fato de os indivíduos apresentarem uma tendência de serem mais altos, sem um aumento proporcional no tamanho da copa, sugere que os jovens de espécies arbóreas estariam investindo predominantemente no crescimento vertical, como forma de "fuga" a essas condições adversas. Já os indivíduos de Arecaceae, são plantas de sub-bosque que apresentam um tamanho de copa muito mais relacionado ao tamanho e expansão de suas folhas. Sendo assim, a partir de uma certa altura, os indivíduos já apresentariam folhas iguais àquelas produzidas por adultos (Carvalho et al. 1999) e, portanto, o aumento lateral deve ser muito maior, conforme o encontrado. Possivelmente, a partir de uma altura maior, essa relação se inverta novamente, visto que as espécies de Arecaceae não apresentam expansão lateral devido às ramificações.

A pequena variação observada, indicada pelo coeficiente de determinação pode estar relacionada às características específicas, sugerindo pequenas diferenças alométricas entre espécies nesse estádio ontogenético inicial, relacionadas às suas histórias filogenéticas. Sterck \& Bongers (2001) observaram que características específicas e diferenças na disponibilidade de luz podem influenciar a arquitetura de espécies arbóreas. Da mesma forma, Nishimura \& Suzuki (2001) também encontraram relações alométricas diferentes para comunidades de plântulas ocupando diferentes tipos de floresta, mas observaram uma convergência na arquitetura das plântulas em cada tipo de floresta. O fato de jovens de diferentes espécies se ajustarem a um único modelo alométrico sugere que os fatores ecológicos podem ter um papel restritivo na alometria das plântulas e jovens, principalmente por todas estarem sujeitas à condições ambientais severas, a despeito de possíveis diferenças alométricas entre espécies, ligadas à restrições filogenéticas.

Portanto, as pressões ecológicas, parecem influenciar a alometria de indivíduos pequenos de espécies arbóreas, podendo ser determinantes da arquitetura dos indivíduos adultos sob diferentes condições ambientais.

\section{Agradecimentos}

Os autores desejam agradecer ao Prof. Dr. Jean Paul Metzger pelas facilidades oferecidas durante este trabalho, a Luciana F. Alves, Bete Canela, Marquito Liesenfeld, Dani Bertani, Cristiano Verola e e ao incansável Renato pela enorme ajuda na coleta de dados, a Luciana F. Alves, Marisa G. Fonseca, Flávio N. Ramos e a dois assesores anônimos pelas importantes sugestões ao manuscrito, a SABESP por permitir o trabalho na Reserva e a FAPESP pelo apoio financeiro (Proc. 00/00596-0).Esta pesquisa fez parte do projeto temático "Consevaçao da Biodiversidade em Paisagens Fragmentadas no Planalto Atlantico de São Paulo" (Fapesp n. 99/05123-4) e constitui a sua publicação n. 1.

\section{Referência bibliográficas}

ALVES, L.F. \& SANTOS, F.A.M. 2002. Tree allometry and crown shape of four tree species in Atlantic rain forest, south-east Brazil. J. Trop. Ecol. 18:245-260.

BOND, W.J., HONIG, M. \& MAZE, K.E. 1999. Seed size and seedling emergence: an allometric relationship and some ecological implications. Oecologia 120:132-136.

CANHAM, C.D. 1988. Growth and canopy architecture of shade-tolerant trees: response to canopy gaps. Ecology 69:786-795.

CARVALHO, R.M., MARTINS, F.R. \& SANTOS, F.A.M. 1999. Leaf ecology of pre-reproductive ontogenetic stages of the palm tree Euterpe edulis Mart. (Arecaceae). Ann. Bot. 83:225-233.

CORNELISSEN, J.H.C. 1999. A triangular relationship between leaf size and seed size among woody species: allometry, ontogeny, ecology and taxonomy. Oecologia 118:248-255. 
DALLING, J.W, LOVELOCK, C.E. \& HUBBELL, S.P. 1999. Growth responses of seedling of two neotropical pioneer species to simulated forest gap environments. J. Trop. Ecol. 15: 827-839.

KIDSON, R. \& WESTOBY, M. 2000. Seed mass and seedling dimensions in relation to seedling establishment. Oecologia 125:11-17.

KING, D.A. 1994. Influence of light level on the growth and morphology of saplings in a Panamanian forest. Am. J. Bot. 811:948-957.

KING, D.A. 1996. Allometry and life history of tropical trees. J. Trop. Ecol. 12:25-44.

KOHYAMA, T. 1987. Significance of architecture and allometry in sapling. Funct. Ecol. 1: 399-404.

KÖPPEN, W. 1948. Climatologia. Fondo de Cultura Económica. México.

NIKLAS, K. J. 1994. Plant allometry: the scaling of form and process. The University of Chicago Press, Chicago.

NISHIMURA, T.B. \& SUZUKI, E. 2001. Allometric differentiation among tropical tree seedlings in heath and peatswamp forests. J. Trop. Ecol. 17:667-681.

OMETTO, J.C. 1981. Bioclimatologia vegetal. Editora Agronômica Ceres Ltda, São Paulo.

SABESP. 1997. Programa de Conservação do Sistema Cotia. Relatório Conclusivo (tomo 3): Avaliação Ambiental. SABESP/Fundação Brasileira para o Desenvolvimento Sustentável, São Paulo.

SPOSITO, T.C. \& SANTOS, F.A.M. 2001. Scaling of stem and crown in eight Cecropia (Cecropiaceae) species of Brazil. Am. J. Bot. 88:939-949.

STERCK, F.J. \& BONGERS, F. 2001. Crown development in tropical rain forest trees: patterns with tree height and light availability. J. Ecol. 89:1-13.

VELOSO, P. H., RANGEL-FILHO, A. L. R. E LIMA, J. C. A. 1991. Classificação da vegetação brasileira adaptada a um sistema universal. IBGE, Rio de Janeiro.

WRIGHT, I.J. \& WESTOBY, M. 2001. Understanding seedling growth relationships through specific leaf area and leaf nitrogen concentration: generalisations across growth forms and growth irradiance. Oecologia 127:2129.

ZAR, J. H. 1999. Biostatistical Analysis. $4^{\text {th }}$ edition. Prentice Hall, New Jersey.
Título: Alometria de plântulas e jovens de espécies arbóreas: copa $\mathrm{x}$ altura

Autores: Rita de Cássia Quitete Portela \& Flavio Antonio Maës dos Santos

Biota Neotropica, Vol. 3 (número 2): 2003

http://www.biotaneotropica.org.br/v3n2/pt/ abstract?article+BN00503022003

recebido em: 30/04/2003

revisaod em: 23/06/2003

publicado em: 01/07/2003

ISSN 1676-0603 